INTESTINE

\title{
Citrulline increases arginine pools and restores nitrogen balance after massive intestinal resection
}

\author{
S Osowska, C Moinard, N Neveux, C Loï, L Cynober
}

Gut 2004;53:1781-1786. doi: 10.1136/gut.2004.042317

See end of article for authors' affiliations

.....................

Correspondence to:

Dr S Osowska, Laboratoire de Biologie de la Nutrition, Faculté de PharmacieUniversité Paris 5, 4 Avenue de l'Observatoire, 75270 Paris Cedex 06, France; moinard@ pharmacie.univ-paris5.fr

Revised version received 3 May 2004

Accepted for publication 11 May 2004
Objective: Arginine supplementation seems logical in situations where this amino acid becomes essential, for example after massive intestinal resection. Arginine is taken up and metabolised by the liver to a large extent and its supplementation is potentially unsafe. Citrulline is not captured by the liver and passes freely to the kidneys where it is metabolised to arginine, and so is a good candidate to generate arginine and thereby improve nutritional status.

Methods: Twenty four rats were assigned to four groups: citrulline, arginine, control, and sham. The sham group underwent transection and the three other groups resection of $80 \%$ of the small intestine. All rats were fed by enteral nutrition and its composition was as follows: supplementation with citrulline in the citrulline group, supplementation with arginine in the arginine group, and standard polymeric enteral nutrition in the control and sham groups. All groups received isonitrogenous nutrition and citrulline and arginine intakes were equimolar in their respective groups. After 10 days, the rats were sacrificed.

Results: Arginine concentration was higher $(p<0.05)$ in plasma and muscle in the citrulline group than in the three other groups. Plasma levels of arginine were 110 (12), 79 (7), 167 (22), and 228 (13) $\mu \mathrm{mol} / \mathrm{l}$ in the sham, control, arginine, and citrulline groups respectively. Arginine concentrations in the gastrocnemius were: 0.15 (0.02), 0.16 (0.02), 0.40 (0.05), and 0.94 (0.20) $\mu \mathrm{mol} / \mathrm{g}$, respectively. Citrulline preserved nitrogen balance in resected rats but not in arginine supplemented rats (mean J10: $2.27(0.29), 1.67(0.15), 1.98(0.29)$, and $2.43(0.41) \mathrm{g} / 24$ hours in the sham, control, arginine, and citrulline groups, respectively).

Conclusion: Supplementing the diet with citrulline is a very efficient means of increasing arginine levels and improving nitrogen balance after massive intestinal resection. The results of this study form a strong rationale for citrulline supplementation in these patients.
A rginine is recognised as one of the most versatile amino acids in cells. ${ }^{1}$ It serves as a precursor for the synthesis not only of proteins but also of nitric oxide, ${ }^{2}$ polyamines, ${ }^{3}$ proline, ${ }^{4}$ and agmatine-a recently discovered decarboxylation product of arginine but whose physiological roles have yet to be established. ${ }^{5}$ Arginine stimulates the secretion of hormones such as insulin, prolactin, glucagon, and growth hormone, ${ }^{6} 7$ and plays a key role in the nitrogen homeostasis modulating ureagenesis. ${ }^{8}$ A balance between arginine intake, arginine production, and its degradation maintains arginine homeostasis.

The liver and gut are two organs that play a major role in the metabolism of arginine. As the activity of arginase is very high in the liver, ${ }^{9}$ most of the arginine that arrives in the liver is converted into urea. If unregulated, this process would be detrimental in certain situations. This is why arginine flux to the liver is modulated by the intestine, which can convert dietary arginine to citrulline. ${ }^{10}$ As the activity of the two enzymes that metabolise citrulline (argininosuccinate synthetase and argininosuccinate lyase) is very low in the intestine, ${ }^{11}{ }^{12}$ citrulline produced by enterocytes cannot be used in situ and citrulline is released as such into the circulation. It is not taken up by the liver but captured by the kidneys. Renal citrulline uptake is approximately $80 \%$ of the amount released by the gut. ${ }^{6}$ In turn, the kidneys generate arginine, which is released into the blood stream for use by other tissues for protein synthesis and other purposes. ${ }^{13}{ }^{14}$ This arginine-citrulline-arginine cycle can be seen as a means of protecting dietary arginine from excessive degradation in the liver, ${ }^{9}$ especially in situations where the intake of protein is low.

In massive intestinal resection, the main site of citrulline production is greatly reduced, and hypocitrullinaemia proportional to the severity of intestinal disease is observed in patients with short bowel syndrome (SBS). ${ }^{15}$ This observation has recently been extended to patients with coeliac disease ${ }^{16}$ and radiation induced small bowel damage. ${ }^{17}$ As a consequence of the diminished plasma levels of citrulline, decreased levels of arginine are also observed, ${ }^{15} 18$ suggesting that arginine becomes an essential amino acid after massive intestinal resection (for example, of rat small intestine). ${ }^{18}$

In this situation, supplementing with arginine would seem logical. However, the fact that arginine is captured and largely metabolised by the liver to yield urea raises questions about the safety of arginine supplementation, which is liable to cause excessive ureagenesis as arginine is the allosteric activator of $\mathrm{N}$-acetylglutamate synthase, which synthesises $\mathrm{N}$-acetylglutamate, which in turn activates carbamoyl-phosphate synthase; hence arginine acts as a catalyst of ureagenesis. ${ }^{19} 20$ On the other hand, citrulline, which is not taken up by the liver and is the major precursor of arginine, should be a good candidate to generate arginine and improve nutritional status in massive intestinal resection. The aim of this work was to test this hypothesis.

\section{MATERIALS AND METHODS \\ Chemicals}

All chemicals were purchased from Sigma (St-QuentinFallavier, France). Citrulline was a gift from Biocodex (Compiègne, France).

Abbreviations: EDL, extensor digitorum longus; SBS, short bowel syndrome 


\section{Animals}

Male Wistar rats ( $\mathrm{n}=24$, body weight 220-230 g) from Iffa Credo (L'Arbresle, France) were used. They were acclimatised in metabolic cages for five days before surgery, with free access to standard laboratory chow ( $17 \%$ protein, 3\% fat, 59\% carbohydrate, $21 \%$ water, vitamins and minerals-A04; Usine d'Alimentation Rationnelle, Villemoisson-sur-Orge, France) and water.

\section{Surgical procedures}

Animals were fasted for 12 hours before surgery. They were anaesthetised by isoflurane inhalation (3\% isoflurane) using a regulated airflow apparatus (Minerve, Esternay, France). Enterectomy was performed on rats in the citrulline, arginine, and control groups (see below for definition) by removing $80 \%$ of the small intestine, leaving $10 \%$ of the proximal jejunum and $10 \%$ of the distal ileum. Six rats were sham operated (transection). For both resected and transected animals, continuity was restored with end to end anastomosis using a single running silk 6-0 suture. Placement of the gastrostomy tube immediately followed intestinal resection or transection. A silicone tube (Tygon, size $0.51 \mathrm{~mm}$; Fisher Bioblock Scientific, Illkirch, France) was introduced into the lumen of the stomach and the remaining end of the catheter was tunnelled subcutaneously to the back of the neck and attached to a spring coil-swivel mechanism (Harvard Apparatus, Les Ulis, France) allowing free movement of the rat in the cage.

One of the authors (CM) is authorised by the French government (No 75522) to use this experimental model of surgery. Animal care complied with French regulations for the protection of animals used for experimental and other scientific purposes (D 2001-486) and with European Community regulations (Official Journal of the European Community, L538 12:18:1986).

\section{Postoperative care}

Rats were housed individually in metabolic cages. They were allowed a five day recovery period. On the first two days they had free access to a 5\% glucose solution. On day 3 glucose was withdrawn and rats had access to standard laboratory chow and water. On day 4 enteral nutrition was introduced at a flow rate of $0.5 \mathrm{ml} / \mathrm{h}$ using a Harvard infusion pump (pump 11; Harvard Apparatus). The rats also had free access to chow food and water. The flow rate was gradually increased to a maximum on day 5 corresponding to intake of $2 \mathrm{~g} \mathrm{~N} / \mathrm{kg} /$ day. From day 5 until the end of the experiment, rats received only enteral nutrition and had free access to water.

\section{Experimental groups}

The rats were randomly assigned to one of four groups (table 1).

- The citrulline group $(n=6)$ consisted of rats receiving standard enteral nutrition (Sondalis; Nestlé Clinical Nutrition, $1 \mathrm{kcal} / \mathrm{ml}$ ) supplemented with citrulline at a dose of $1 \mathrm{~g} / \mathrm{kg} /$ day $(5.708 \mathrm{mM} / \mathrm{kg} /$ day $)$.
- The arginine group $(n=6)$ consisted of rats receiving standard enteral nutrition supplemented with arginine at a dose of $0.994 \mathrm{~g} / \mathrm{kg} /$ day, which was equimolar to the citrulline group.

- The control group $(n=6)$ and the sham group $(n=6)$ consisted of rats receiving standard enteral nutrition.

The standard enteral diet contained $5.8 \mathrm{~g}$ of arginine/100 $\mathrm{g}$ protein, providing an intake of $0.746 \mathrm{~g}$ of arginine $/ \mathrm{kg} / \mathrm{day}$.

To keep all groups isonitrogenous, standard enteral nutrition in the citrulline, control, and sham groups were supplemented with a mixture of non-essential amino acids consisting of alanine, asparagine, glycine, serine, histidine, and proline in equimolar amounts. All groups were isonitrogenous (2 g N/kg/day) and isocaloric (200 kcal/kg/day) (see table 1).

Rats were weighed and urine was collected daily. Total enteral nutrition was administered for 10 days. Enteral nutrition was stopped two hours before decapitation.

\section{Tissue removal}

Blood

Blood was sampled in heparinised tubes, which were rapidly centrifuged.

\section{Liver}

The abdominal cavity was opened. The liver was immediately removed and weighed, and a sample was cut off, frozen in liquid nitrogen, and stored at $-80^{\circ} \mathrm{C}$.

\section{Jejunum and ileum}

The intestinal mucosa was washed with cold $\mathrm{NaCl}(0.9 \%)$, reverted and scraped, rapidly frozen in liquid nitrogen, and stored at $-80^{\circ} \mathrm{C}$ until analysis.

\section{Muscles}

Three muscles of the hindlimbs (extensor digitorum longus (EDL), gastrocnemius, and soleus) were rapidly removed, weighed, frozen in liquid nitrogen, and stored at $-80^{\circ} \mathrm{C}$ until analysis. These three muscles were selected because they differ widely in their function and fibre type ${ }^{21}$ and have different metabolic responses to stress. ${ }^{22} 23$

\section{Parameters studied and analytical methods Nitrogen}

Nitrogen was quantified by chemiluminescence ${ }^{24}$ using an Antek 9000 apparatus (Antek, Houston, Texas, USA) and nitrogen balance was calculated as the difference between nitrogen intake and nitrogen urinary output.

\section{Amino acid concentrations in plasma and tissues (muscles, liver, and intestinal mucosa).}

Plasma was deproteinised with a $30 \%(\mathrm{w} / \mathrm{v})$ sulphosalicylic acid solution. Supernatants were stored at $-80^{\circ} \mathrm{C}$ until analysis. Tissues were ground and deproteinised with $10 \%$ trichloroacetic acid containing 0.5 mM EDTA. ${ }^{25}$ Supernatants

Table 1 Caloric and nitrogen intake in the four groups under study

\begin{tabular}{|c|c|c|c|c|c|c|c|c|c|c|}
\hline \multirow[b]{2}{*}{ Group } & \multirow{2}{*}{$\begin{array}{l}\text { Calories } \\
\text { (kcal/kg/24 h) }\end{array}$} & \multirow{2}{*}{$\begin{array}{l}\text { Nitrogen } \\
(\mathrm{g} / \mathrm{kg} / 24 \mathrm{~h})\end{array}$} & \multicolumn{8}{|c|}{ Supplement (g/kg/24 h) } \\
\hline & & & Cit & Arg & Ala & Asn & Gly & Ser & His & Pro \\
\hline Citrulline & 200 & 2 & 1.000 & - & 0.085 & 0.063 & 0.071 & 0.01 & 0.049 & 0.109 \\
\hline Arginine & 200 & 2 & - & 0.994 & - & - & - & - & - & - \\
\hline Control & 200 & 2 & - & - & 0.339 & 0.251 & 0.286 & 0.399 & 0.197 & 0.438 \\
\hline Sham & 200 & 2 & - & - & 0.339 & 0.251 & 0.286 & 0.399 & 0.197 & 0.438 \\
\hline
\end{tabular}

See materials and methods for more details.

Cit, citrulline; Arg, arginine; Ala, alanine; Asn, asparagine; Gly, glycine; Ser, serine; His, histidine; Pro, proline. 
Table 2 Weight of the organs in the four groups

\begin{tabular}{|c|c|c|c|c|}
\hline Organ & Sham & Control & Arginine & Citrulline \\
\hline Liver (g) & $9.61(0.21)$ & $9.43(0.60)$ & $8.75(0.41)$ & $8.87(0.35)$ \\
\hline Gastrocnemius (g) & $1.31(0.02)$ & $1.28(0.11)$ & $1.10(0.04)$ & $1.16(0.02)$ \\
\hline Soleus (mg) & $104(5)$ & $88(12)$ & $97(4)$ & $98(6)$ \\
\hline $\mathrm{EDL}(\mathrm{mg})$ & $119(9)$ & $113(6)$ & $114(3)$ & $118(3)$ \\
\hline Jejunum $(\mathrm{mg} / \mathrm{cm})$ & $52(6)^{*}$ & $131(18) \dagger$ & $130(17) \dagger$ & $122(13) \dagger$ \\
\hline lleum $(\mathrm{mg} / \mathrm{cm})$ & $38(2)^{*}$ & $129(12) \dagger$ & $110(8) \dagger$ & $122(12) \dagger$ \\
\hline \multicolumn{5}{|c|}{$\begin{array}{l}\text { Results are presented as mean (SEM). } \\
\text { Values in a row with different symbols are statistically different. } \\
\text { EDL extensor diaitorum lonqus. }\end{array}$} \\
\hline
\end{tabular}

were stored at $-80^{\circ} \mathrm{C}$ until analysis. Amino acids were measured by ion exchange chromatography using an amino acid auto analyser (model Aminotac; Jeol, Tokyo, Japan). The results of our participation in the European Quality Control Scheme (ERNDIM) indicate the accuracy of our amino acid determinations.

\section{Statistical analysis}

Data are expressed as means (SEM). Comparisons between sets of data were made using one way analysis of variance (ANOVA) followed by the Newman-Keuls test. PCSM software was used (Deltasoft, Grenoble, France). Differences at $\mathrm{p}<0.05$ were considered significant.

\section{RESULTS}

The body weight gain (the difference between body weight on the first day of total enteral nutrition administration and the last day of the experiment) did not differ significantly in the four groups (data not shown).

Regarding tissue weight (table 2), we found no significant differences in muscle and liver weights in the four groups. As a result of intestinal adaptation, the weight of intestinal mucosa (expressed in $\mathrm{g} / \mathrm{cm}$ ) was significantly higher in the three resected groups than in the sham group (citrulline, arginine, control $v$ sham; $\mathrm{p}<0.05$ ). However, there was no significant difference in intestinal mucosa weight in the three resected groups.

\section{Amino acid concentrations}

Citrulline, arginine, and ornithine were the only amino acids whose profiles were influenced by the different diets.

\section{Plasma (fig 1)}

Intestinal resection caused decreased levels of citrulline compared with the sham group (control $v$ sham; $\mathrm{p}<0.05$ ). Supplementation with arginine raised the concentration of citrulline to levels of the sham group but were still not significantly different from controls. Supplementation with citrulline raised levels of citrulline to significantly higher values than in the other three groups (citrulline $v$ sham, control, arginine; $\mathrm{p}<0.05$ ). Plasma ornithine concentrations were increased in the arginine and citrulline groups compared with the sham and control groups (citrulline and arginine $v$ sham and control; $\mathrm{p}<0.05$ ).

The plasma level of arginine decreased $(-28 \%)$ in controls compared with the sham group but the difference did not reach significance. Addition of arginine to the diet raised its concentration to significantly higher values compared with both controls and the sham group (arginine $v$ sham and control; $\mathrm{p}<0.05)$. The concentration of arginine in the citrulline supplemented group was significantly higher than in all other groups (citrulline $v$ arginine, control, and sham; $\mathrm{p}<0.05$ ).

\section{Intestinal mucosa (table 3)}

In the jejunum, arginine concentrations in controls were significantly lower than in the sham group (control $v$ sham; $\mathrm{p}<0.05$ ). Supplementation of arginine or citrulline raised arginine levels to values comparable with those in the sham group (arginine and citrulline $v$ control; $\mathrm{p}<0.05$ ).

Jejunal and ileal citrulline concentrations were increased in the citrulline compared with the three other groups (citrulline $v$ sham, control, arginine; $\mathrm{p}<0.05$ ).

\section{Liver (table 4)}

Citrulline supplementation did not result in its accumulation. There was an increase in ornithine in the citrulline supplemented group (citrulline $v$ sham, control; $\mathrm{p}<0.05$ ). Free arginine was not detected in any group.

\section{Muscles (table 5)}

Levels of the three amino acids were unchanged in controls compared with the sham group. Arginine supplementation
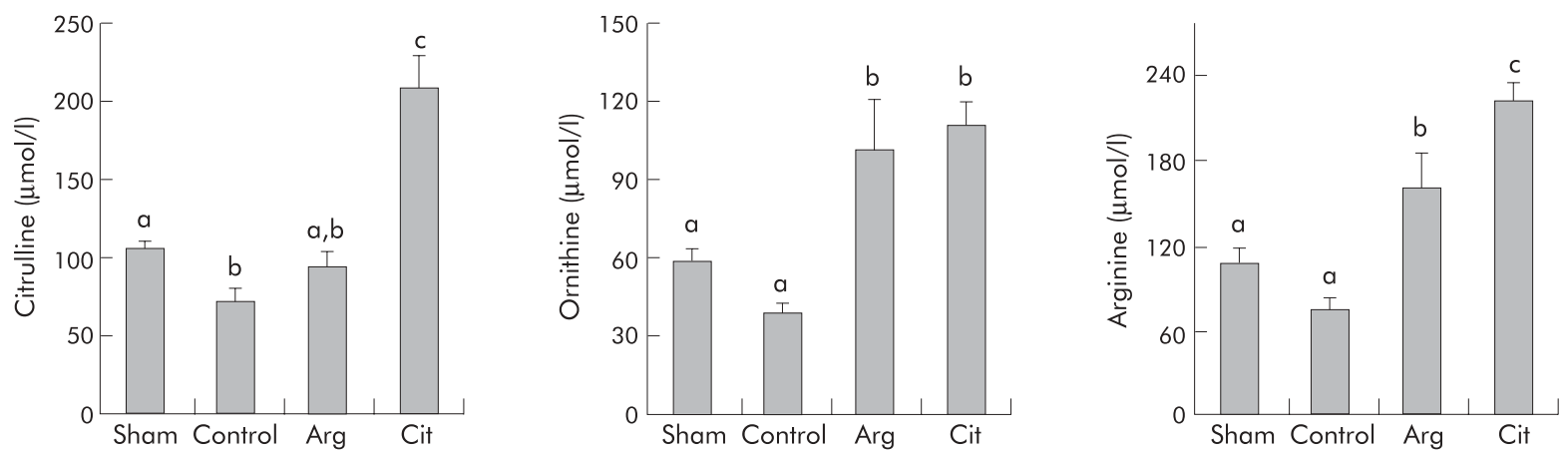

Figure 1 Plasma citrulline, ornithine, and arginine concentrations at the end of the enteral nutrition programme. Four groups of rats were studied: sham (transected rats fed standard enteral nutrition), control (resected rats fed standard enteral nutrition), arginine (Arg; resected rats fed arginine supplemented diet), citrulline (Cit; resected rats fed citrulline supplemented diet). Results are expressed as mean values (SEM). Values with different superscript letters are statistically different. 
Table 3 Amino acid concentrations in the intestinal mucosa in the four groups

\begin{tabular}{|c|c|c|c|c|}
\hline & Sham & Control & Arginine & Citrulline \\
\hline \multicolumn{5}{|c|}{ Jejunum $(\mathrm{nmol} / \mathrm{g}$ ) } \\
\hline Cit & $285(30)^{*}$ & $180(47)^{*}$ & $365(50)^{*}$ & $756(117)+$ \\
\hline Orn & $57(7)^{*}$ & $52(6)^{*}$ & $72(7)^{*}+$ & $83(8) t$ \\
\hline Arg & $523(127)^{*}$ & $172(19) t$ & $535(78)^{*}$ & $585(98)^{*}$ \\
\hline \multicolumn{5}{|c|}{ lleum $(\mathrm{nmol} / \mathrm{g}$ ) } \\
\hline Cit & $252(50)^{*}$ & $205(41)^{*}$ & $385(78)^{*}$ & $613(89) \dagger$ \\
\hline Orn & $48(6)$ & $38(7)$ & $82(32)$ & $77(10)$ \\
\hline Arg & $702(247)$ & 278 (124) & $638(123)$ & $527(72)$ \\
\hline
\end{tabular}

raised levels of ornithine in the soleus compared with the sham group (arginine $v$ sham; $<<0.05$ ) and in the soleus and EDL compared with the control group (arginine $v$ control; $\mathrm{p}<0.05$ ). The effect on arginine concentration of the arginine supplement was seen only in the soleus where arginine levels were significantly higher than in both the sham and control groups (arginine $v$ sham and control; $\mathrm{p}<0.05$ ). Citrulline supplementation raised levels of citrulline in the three muscles so that they differed significantly from the other three groups (citrulline $v$ sham, control, and arginine; $\mathrm{p}<0.05)$. Levels of ornithine were higher in the gastrocnemius and soleus in the citrulline group compared with the sham and control groups (citrulline $v$ sham and control; $\mathrm{p}<0.05$ ). Arginine levels in all muscles were significantly higher in the citrulline group than in the other three groups (citrulline $v$ sham, control, and arginine; $\mathrm{p}<0.05$ ).

\section{Nitrogen balance (fig 2)}

For the first four days of the experiment there was no significant change in nitrogen balance in the groups. Starting from day 6, nitrogen balance in the control group fell compared with the sham and citrulline groups (control $v$ sham and citrulline; $\mathrm{p}<0.05$ ). This value did not change up to the end of the experiment.

Arginine supplementation did not restore nitrogen balance which remained diminished on days 9 and 10 compared with sham and citrulline (arginine $v$ sham and citrulline; $\mathrm{p}<0.05$ ). On day 10, however, it differed significantly from controls (arginine $v$ control; $\mathrm{p}<0.05$ ).

The effect of citrulline supplementation was seen very early: starting from day 5 it restored nitrogen balance compared with controls (citrulline $v$ control; $\mathrm{p}<0.05$ ) and from day 7 compared with the arginine group (citrulline $v$ arginine; $\mathrm{p}<0.05$ ). It did not differ from the sham group at any time during the experiment.

\section{DISCUSSION}

Although arginine is considered a non-essential amino acid, certain situations lead to increased needs for this amino acid, and de novo synthesis of arginine may not be sufficient to meet these needs. Arginine then becomes an essential amino acid. Such situations include growth, ${ }^{26}$ hypermetabolic situations such as burn injury, ${ }^{27} 28$ and SBS. ${ }^{18}$

The work of Wakabayashi and colleagues ${ }^{18}{ }^{29}$ recognised arginine as an essential amino acid by showing that removing arginine from the diet after resection led to diminished levels of muscle arginine and negative nitrogen balance. Our experimental conditions were different, as the standard enteral nutrition formula used contained arginine (see materials and methods) and differences between groups were due to supplementation. To our knowledge, use of an arginine supplemented diet in SBS has not previously been studied and our work is therefore the first to show how it influences nitrogen balance and plasma and muscle levels of arginine. In our study, the arginine supplement raised arginine levels in plasma by $38 \%$ compared with the sham group. In healthy animals, a $2 \%$ arginine supplement in drinking water raises arginine levels to twice those of nonsupplemented animals. ${ }^{3031}$ This suggests that the body demand for arginine in the situation of intestinal resection is elevated, and this demand is only partially met by arginine supplementation. Similarly, nitrogen balance was also only partially restored by arginine supplementation and it differed from the control group only on the last day of the experiment. It remained however diminished compared with the sham group throughout the experiment.

A large part of dietary arginine is metabolised in enterocytes to yield citrulline. It has been shown that the intestine is the main site of citrulline synthesis ${ }^{10}$ and this was confirmed in our work by decreased plasma citrulline concentrations in the resected group of rats fed the nonsupplemented diet. Unlike arginine, which is extensively taken up by the liver and metabolised to urea, intestine derived citrulline passes freely through the liver. This is well supported in the present study by the fact that citrulline content in the liver did not vary in the citrulline supplemented group whereas there was large accumulation of citrulline in all of the other tissues under study. Citrulline is then captured by the kidneys. Eighty per cent of citrulline synthesised by the intestine is converted into arginine, ${ }^{6}$ which is released into the blood stream for use by other tissues for protein synthesis and other purposes..$^{13}{ }^{14}$ Considering the metabolic features of arginine (partial restoration of nitrogen balance, low increase in plasma arginine, and lack of increase in gastrocnemius and EDL after arginine supplement) we conclude that the remnant intestine is unable to convert dietary arginine to citrulline adequately, and that a large part of arginine supplementation is taken up and metabolised by the liver. This limits the efficacy of arginine supplementation in intestinal resection.

Table 4 Amino acid concentrations in the liver in the four groups

\begin{tabular}{lllcl}
\hline & Sham & Control & Arginine & Citrulline \\
\hline Citrulline $(\mathrm{nmol} / \mathrm{g})$ & $110(20)$ & $110(30)$ & $90(30)$ & $110(30)$ \\
Ornithine $(\mathrm{nmol} / \mathrm{g})$ & $350(30)^{*}$ & $350(60)^{*}$ & $570(90)^{*} \dagger$ & $760(80) \dagger$ \\
\hline
\end{tabular}

Results are expressed as mean values (SEM)

Values in a row with different symbols are statistically different. 


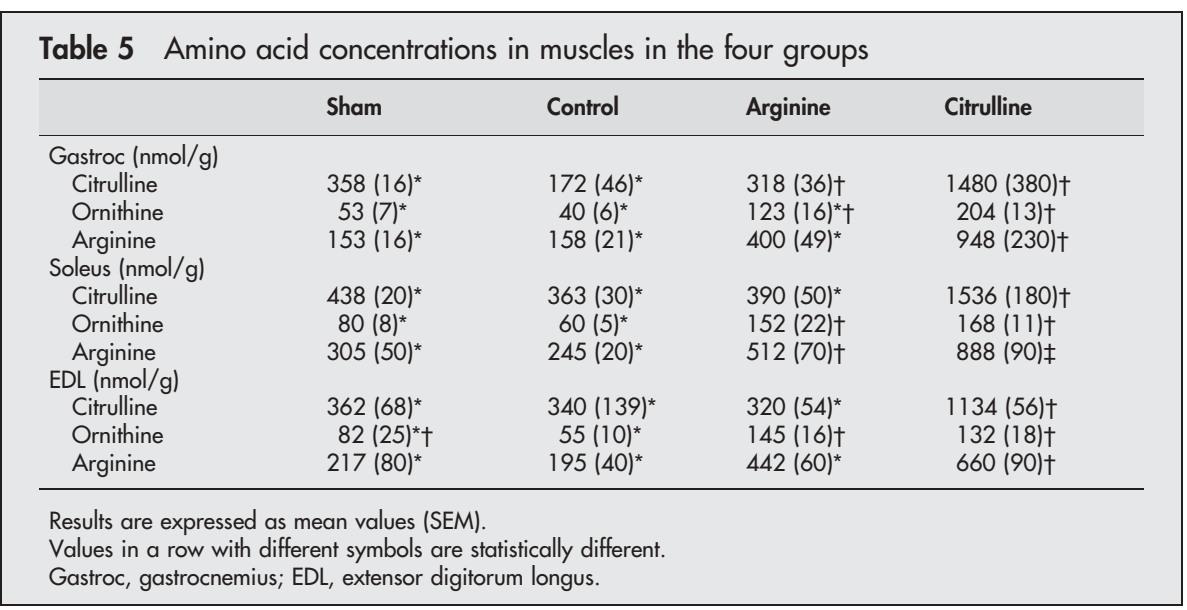

Inefficiency of an arginine supplement in a situation where the intestinal synthesis of citrulline was inhibited was studied by Hoogenraad and colleagues. ${ }^{32}$ Using a selective inhibitor of intestinal ornithine transcarbamylase, an enzyme that synthesises citrulline from ornithine and carbamoylphosphate, they showed that rats fed on normal rat chow, with presumably a sufficient intake of arginine, lost weight. Addition of arginine only partially restored weight gain. Serum arginine levels were also reduced by $25 \%$ and restored by addition of $1 \%$ citrulline.

The fact that citrulline is not taken up by the liver suggests that it may be a good means of increasing arginine levels in intestinal resection. Our experiment shows that citrulline raises arginine levels in plasma and all muscles much more effectively than arginine itself. Even at the intestinal level, citrulline can generate the same amounts of arginine as arginine itself. The effect of arginine or citrulline supplementation on the structure of the intestine of the resected rats was not studied here, but may be of major importance as it has been shown that arginine supplementation has a positive effect resulting in increased villus height and crypt cell mitoses. ${ }^{33}$ This point deserves further research.

Unlike arginine supplementation, which had no positive effect on nitrogen balance, citrulline supplement restored nitrogen balance, keeping it at the level of the sham group throughout the experiment. No attempt was made to measure nitrogen in stools, and this may be a limitation of our study, but there is no reason to believe that nitrogen loss in stools would be different in the three groups of rats with SBS. In addition, it is noteworthy that stool volume was measured in rats under enteral nutrition and no rat in any of the groups developed diarrhoea at any time during the experiment.

Although the differences in nitrogen balance between groups were obvious, this was not reflected in body weight gain. In order to solve this apparent conflict, it would be of interest to measure body composition of rats and to evaluate fat free mass.

Taken as a whole, our results show that citrulline, by generating large amounts of arginine and totally restoring nitrogen balance, can be an important supplement to a diet of patients who have undergone massive intestinal resection. It is tempting to speculate whether citrulline, although not a component amino acid of proteins, can influence protein synthesis and become an essential amino acid in intestinal resection. Assessment of protein synthesis would be necessary to determine this. Further work to evaluate the fate of the generated arginine would also be valuable to investigate the beneficial effect of citrulline supplementation more fully.

In addition, it would be of great interest to compare the behaviour and effects of arginine and citrulline when administrated by the parenteral route. Providing arginine

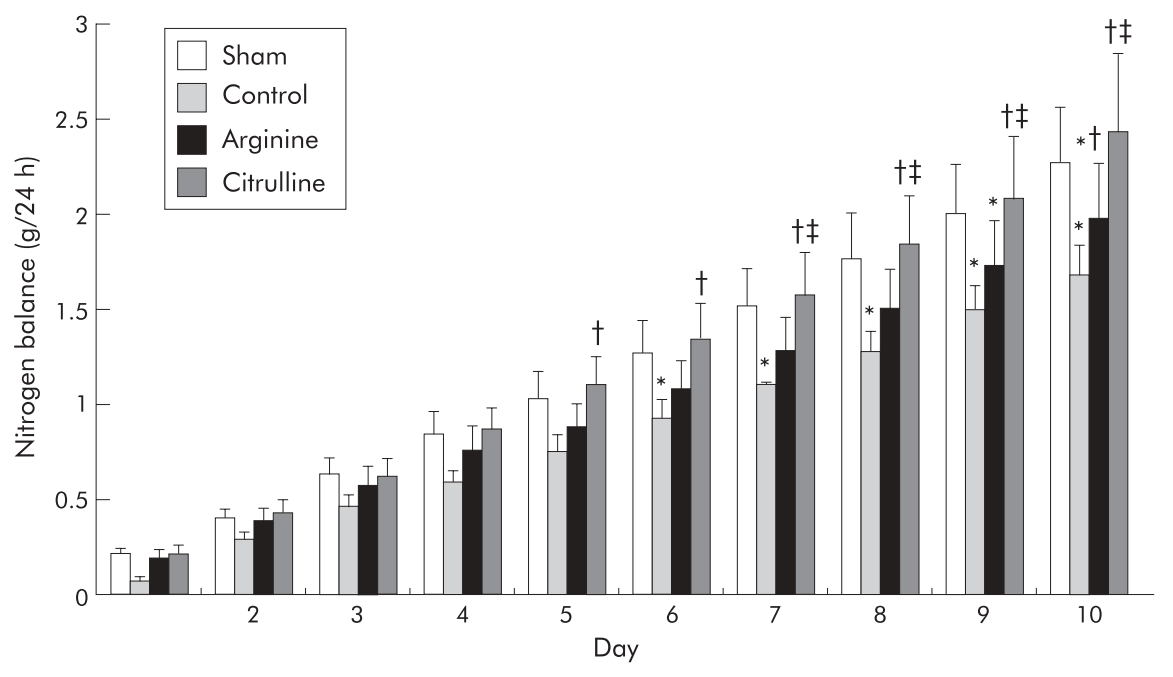

Figure 2 Nitrogen balance during 10 days of total enteral nutrition. Four groups of rats were studied: sham (transected rats fed standard enteral nutrition), control (resected rats fed standard enteral nutrition), arginine (resected rats fed arginine supplemented diet), and citrulline (resected rats fed citrulline supplemented diet). Results are expressed as the difference between daily nitrogen intake and daily urinary nitrogen excretion. Results are presented as mean values (SEM). * $p<0.05$ versus sham, $\uparrow p<0.05$ versus control, $\neq p<0.05$ versus arginine. 
by the parenteral route would bypass splanchnic extraction and in this case arginine could be more efficient than when provided enterally. This point deserves further study as in postoperative care, patients with SBS are fed by the parenteral route. ${ }^{34}$ Finally, it would be interesting to extend the study of the effects of citrulline to other situations of intestinal insufficiency, such as Crohn's disease or necrotising enterocolitis.

\section{ACKNOWLEDGEMENTS}

This work was supported by a contrat quadriennal grant from the French Ministry of Research and Technology. S Osowska was the recipient of a fellowship from Laboratoires Biocodex. We thank Harvard Apparatus and Minerve for providing devices used in this study.

\section{Authors' affiliations}

S Osowska, C Moinard, C Loï, Laboratoire de Biologie de la Nutrition, EA 2498, Faculté de Pharmacie-Université Paris 5, Paris, France N Neveux, L Cynober, Laboratoire de Biologie de la Nutrition, EA 2498, Faculté de Pharmacie-Université Paris 5, Paris, France, and Laboratoire de Biochimie, Hôtel-Dieu, AP-HP, Paris, France

\section{REFERENCES}

1 Wu G, Morris SM Jr. Arginine metabolism: nitric oxide and beyond. Biochem J 1998:336:1-17.

2 Hibbs JB Jr, Taintor RR, Vavrin Z. Macrophage cytotoxicity: role for L-arginine deiminase and imino nitrogen oxidation to nitrite. Science 1987;235:473-6.

3 Seiler N, Heby O. Regulation of cellular polyamines in mammals. Acta Biochim Biophys Hung 1988;23:1-35.

4 Welters $\mathrm{CF}$, Dejong $\mathrm{CH}$, Deutz $\mathrm{NE}$, et al. Effects of parenteral arginine supplementation on the intestinal adaptive response after massive small bowel resection in the rat. J Surg Res 1999;85:259-66.

5 Morris SM. Regulation of arginine availability and its impact on NO synthesis. In: Ignarro $\sqcup$, eds. Nitric oxide: biology and pathobiology. New York: Academic Press, 2000:187-97.

6 Barbul A. Arginine: biochemistry, physiology, and therapeutic implications. JPEN J Parenter Enteral Nutr 1986;10:227-38

7 Reyes AA, Karl IE, Klahr S. Role of arginine in health and in renal disease. Am J Physiol 1994;267:F331-46.

8 Cynober L, Le Boucher J, Vasson MP. Arginine metabolism in mammals. J Nutr Biochem 1995;6:402-13.

9 Cynober L. Can arginine and ornithine support gut functions? Gut 1994:35(suppl):S42-5.

10 Windmueller HG, Spaeth AE. Source and fate of circulating citrulline. Am J Physiol 1981;241:E473-80.

11 Ratner S, Murakami-Murofushi K. A new radiochemical assay for argininosuccinase with purified [14C]argininosuccinate. Anal Biochem 1980;106:134-47.

12 Ratner S. A radiochemical assay for argininosuccinate synthetase with [U14C]aspartate. Anal Biochem 1983;135:479-88
13 Dhanakoti SN, Brosnan JT, Herzberg GR, et al. Renal arginine synthesis: studies in vitro and in vivo. Am J Physiol 1990;259:E437-42.

14 Featherston WR, Rogers QR, Freedland RA. Relative importance of kidney and liver in synthesis of arginine by the rat. Am J Physiol 1973;224:127-9.

15 Crenn $\mathbf{P}$, Coudray-Lucas $C$, Thuillier $F$, et al. Postabsorptive plasma citrulline concentration is a marker of absorptive enterocyte mass and intestinal failure in humans. Gastroenterology 2000;119:1496-505

16 Crenn P, Vahedi K, Lavergne-Slove A, et al. Plasma citrulline: A marker of enterocyte mass in villous atrophy-associated small bowel disease. Gastroenterology 2003;124:1210-19.

17 Lutgens LC, Deutz NE, Gueulette J, et al. Citrulline: a physiologic marker enabling quantitation and monitoring of epithelial radiation-induced small bowel damage. Int J Radiat Oncol Biol Phys 2003;57:1067-74.

18 Wakabayashi Y, Yamada E, Yoshida T, et al. Arginine becomes an essential amino acid after massive resection of rat small intestine. J Biol Chem 1994;269:32667-71

19 Shigesada K, Tatibana M. N-Acetylglutamate synthetase from rat-liver mitochondria. Partial purification and catalytic properties. Eur J Biochem 1978;84:285-91.

20 Shigesada K, Tatibana M. Enzymatic synthesis of acetylglutamate by mammalian liver preparations and its stimulation by arginine. Biochem Biophys Res Commun 1971;44:1117-24.

21 Maltin CA, Delday MI, Baillie AG, et al. Fiber-type composition of nine rat muscles. I. Changes during the first year of life. Am J Physiol 1989;257:E823-7.

22 Hasselgren PO, Hall-Angeras M, Angeras $U$, et al. Regulation of total and myofibrillar protein breakdown in rat extensor digitorum longus and soleus muscle incubated flaccid or at resting length. Biochem J 1990;267:37-44.

23 James JH, Hasselgren PO, King JK, et al. Intracellular glutamine concentration does not decrease in all muscles during sepsis. J Surg Res 1993;54:558-64.

24 Grimble GK, West MF, Acuti AB, et al. Assessment of an automated chemiluminescence nitrogen analyzer for routine use in clinical nutrition. JPEN J Parenter Enteral Nutr 1988;12:100-6.

25 Le Boucher J, Charret C, Coudray-Lucas C, et al. Amino acid determination in biological fluids by automated ion-exchange chromatography: performance of Hitachi L-8500A. Clin Chem 1997;43:1421-8.

26 Wu G, Meininger CJ, Knabe DA, et al. Arginine nutrition in development, health and disease. Curr Opin Clin Nutr Metab Care 2000:3:59-66.

27 Yu YM, Ryan CM, Burke JF, et al. Relations among arginine, citrulline, ornithine, and leucine kinetics in adult burn patients. Am J Clin Nutr 1995;62:960-8.

28 Yu YM, Ryan CM, Castillo L, et al. Arginine and ornithine kinetics in severely burned patients: increased rate of arginine disposal. Am J Physiol Endocrinol Metab 2001;280:E509-17.

29 Wakabayashi Y, Yamada E, Yoshida T, et al. Effect of intestinal resection and arginine-free diet on rat physiology. Am J Physiol 1995;269:G313-18.

30 Brandes RP, Brandes S, Boger RH, et al. L-arginine supplementation in hypercholesterolemic rabbits normalizes leukocyte adhesion to nonendothelial matrix. Life Sci 2000;66:1519-24.

31 Cooke JP, Singer AH, Tsao P, et al. Antiatherogenic effects of L-arginine in the hypercholesterolemic rabbit. J Clin Invest 1992;90:1168-72.

32 Hoogenraad N, Totino N, Elmer $\mathrm{H}$, et al. Inhibition of intestinal citrulline synthesis causes severe growth retardation in rats. Am J Physiol 1985;249:G792-9.

33 Ozturk H, Dokucu Al, Yagmur Y, et al. Effects of supplemental L-arginine on the intestinal adaptive response after massive small-bowel resection in rats. Pediatr Surg Int 2002;18:332-6.

34 Vanderhoof JA, Langnas AN. Short bowel syndrome in children and adults. Gastroenterology 1997;113:1767-78.

\section{Call for papers}

10th European Forum on Quality Improvement in Health Care

13-15 April 2005, ExCel, Docklands, London

For further information on how to submit your paper please go to:

http://www.quality.bmipg.com 